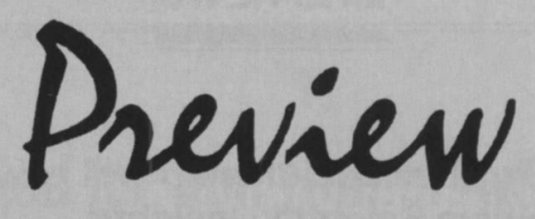

\title{
1992 Spring Meeting
}

\section{April 27 - May 1 - San Francisco, Marriott Hotel}

\author{
Meeting Chairs: \\ June Passaretti, Pfizer, Inc. \\ Lynn Rehn, Argonne National Laboratory \\ Dale Schaefer, Sandia National Laboratories
}

The Spring MRS Meeting returns to San Francisco this year, where it will stay for the decade. While the location is set, the program continues to grow and change. This year's program includes 26 symposia and has 2,400 oral and poster presentations planned, substantially more than any previous spring meeting.

A cluster of symposia will address environmental concerns. One symposium covers recycling of wood-based materials, encompassing recycling paper, removing ink and contaminates, and creating composite structures using recycled fiber. The second symposium of this cluster covers materials for alternative energy sources, such as chemically selective membranes and catalysts, and materials for hightemperature and high-pressure energy conversion. The third symposium addresses materials separation using membranes, zeolites, etc. to handle toxic waste, remove metals, or filter gases.

Fullerenes, fullerides, and fulleroids settle into a symposium on novel forms of carbon, joined by diamond films, carbon clusters, fibers, amorphous carbon, graphite, extraterrestrial carbon, and foams, bringing together diverse interests but focusing on a common element.

The largest symposium will consider "better ceramics through chemistry." As science looks to build unique materials from the atom up, more attention is focusing on the versatility and insight gained through chemistry.

A symposium on "smart materials" and micro-electro-mechanical systems teases the imagination with a vision of tiny modern machines performing tasks by sensing and responding to light, chemistry, temperature, and even biological stimuli.
As computers grow in complexity and capability, so too do computational methods to explain and predict structure, properties, and other materials phenomena. A symposium addressing computational methods will cover modeling of polymers, ceramics, superconductors, interfaces, clusters, processing, and more.

Semiconductors are broadly represented in a series of symposia, including two new ones on photo-induced space charge effects and defect engineering. Additional symposia cover surface preparation, reliability, metallization, heteroepitaxy, beam interactions, and electronic packaging.

Among other intriguing topics is one within the symposium on art and archaeology. Several sessions address the destruction of cultural property and historic monuments resulting from armed conflict and the conservation science required to preserve them. Examples draw from World War I and II, the Persian Gulf War, and recent conflicts surrounding Yugoslavia.

Other symposia cover microwave processing, clusters and colloids, aerosols, intermetallic matrix composites, submicron multiphase materials, defects in oxides, and macromolecular host-guest complexes. See the matrix on the following pages for a list of all the technical symposia and session titles.

\section{Special Features}

The plenary speaker on Monday night will be Bassam Z. Shakhashiri, professor of chemistry at the University of WisconsinMadison. Shakhashiri founded the University of Wisconsin's Institute for Chemical Education in 1983 and was As- sistant Director of the National Science Foundation for Science and Engineering Education from 1984 to 1990 . He is known for his effective teaching methods using demonstrations. He aims both to lure future generations to careers as researchers, entrepreneurs, and teachers and to promote scientific literacy for all citizens. The session also includes presentation of the Outstanding Young Investigator awards and graduate student awards.

Symposium $X$, a set of lunch-hour reviews designed for the nonspecialist, promises enlightening presentations on porous materials, ceramic membranes, art and archaeology, computer simulation of microstructure, mechanical properties of thin films, new optics for $x$-rays and neutrons with the Kumakhov lens, and presentations by the Outstanding Young Investigator awardees.

A special forum is planned in response to the Bush administration's Advanced Materials and Processing Program, an initiative proposed in the 1993 federal budget. Representatives from the major federal agencies affected will describe their involvement in the intiative. The session is tentatively scheduled for noon on Thursday, April 30.

The meeting is complemented by short courses related to symposium topics, an extensive equipment exhibit, a job placement bulletin board, three evenings of poster sessions, and more.

For further details about the meeting program and registration, see the 1992 MRS Spring Meeting Program, which is mailed to all MRS members. If you need a program, call the MRS Meetings Department (412)367-3003; fax (412)367-4373. 


\section{Plenary Speaker}

\section{Bassam Z. Shakhashiri \\ Monday, April 27, 1992 \\ San Francisco Marriott}

An outspoken advocate of science and technology education and literacy, Bassam Z. Shakhashiri is a professor of chemistry at the University of Wisconsin-Madison and holds $\mathrm{AB}, \mathrm{MS}$, and $\mathrm{PhD}$ degrees in chemistry as well as several honorary doctorates. He founded the University of Wisconsin's Institute for Chemical Education in 1983 and is co-author of several texts and videotapes on chemistry. He was the National Science Foundation's assistant director for science and engineering education from 1984 to 1990 and clains credit for having set the NSF education budget on a $\$ 600$ million trajectory for fiscal year 1993.

Shakhashiri is well-known for his development and use of demonstrations in teaching to prove that "science is fun." $\mathrm{He}$ has had an interactive chemistry exhibit on display since 1983 at the Chicago Museum of Science and Industry and annually puts on a Christmas science show that has been presented at various places, including the National Academy of Sciences and the Smithsonian's National Air and Space Museum.

\section{Travel and Lodging}

Meeting Hotel:

San Francisco Marriott Hotel

55 Fourth Street

San Francisco, CA 94103

(800) 228-9290 Nationwide

(415) 896-1600 Direct

FAX (415) 442-0141

A block of rooms has been reserved for MRS meeting attendees at the San Francisco Marriott Hotel. When reserving your room, mention the Materials Research Society to receive the special rates: $\$ 140$ single; $\$ 165$ double.

\section{DEADLINE FOR HOTEL}

RESERVATIONS: March 30, 1992

\section{Air Travel:}

American Airlines is offering special rates for traveling to and from the San Francisco meeting from Friday, April 24, through Monday, May 4, 1992:

$45 \%$ off full-day coach fare (U.S. only), $5 \%$ off all other fares with all tariff rules in effect.

To take advantage of these discounts available only through American Airlines' toll- free number:

1. Call American Airlines today, or have your travel agent call: (800) 433-1790

2. Refer to Star Number: S02Z2VO

\section{Preregistration Fees}

Preregistration fees for the MRS meeting. are $\$ 225$ for MRS members; $\$ 260$ for nonmembers; $\$ 60$ for student members; $\$ 70$ for student nonmembers; and $\$ 95$ for MRS short course attendees registered for two or more short course days.

Preregister by April 17, 1992, to take advantage of pre-meeting fees. Registrations received after April 17, 1992, will be charged at-meeting rates. At-meeting registration fees will be $\$ 50$ higher $(\$ 10$ higher for students) than preregistration fees.

Preregistrations are accepted by mail, phone, or fax. If you need a form for mail or fax preregistration, call (412) 367-3003.

Telephone Preregistrations require credit card payment (VISA, MasterCard, or Diners Club only). Call (412) 367-3003 and ask for Meeting Registration, Monday through Friday between 8:00 a.m. and 5:00 p.m. EST.

Telephone preregistrations will close at 5:00 p.m., Friday, April 17, 1992.

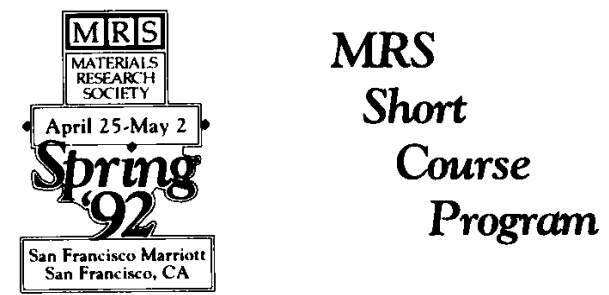

Five New Course Topics and Tutorial

Selected short courses and a tutorial covering the latest developments in materials science and technology will be offered in conjunction with the 1992 Spring Meeting of the Materials Research Society. These up-to-date presentations are at the forefront of science and technology and complement Spring Meeting symposium topics. SPECIALITY, REVIEW, AND SURVEYCOURSES and the TUTORIAL are designed to meet the needs of professional scientists, engineers, professional staff and managers who want to know the latest techniques relating to materials science and technology. For information regarding registration, student scholarships, and special meeting registration discounts, contact MRS Headquarters: Telophone (412) 367-3003; Fax (412) 367-4373.

\section{Advanced Materials}

Optoelectronic Materials, Processes, and Devices

Preregistration Tuition

Instructor: Mool C. Gupta

Friday-Saturday, May 1-2

Polymers for Electronic and Photonic Applications

Instructors: C.P. Wong, C. Grant Willson and Robert J. Twieg

Saturday-Monday morning, April 25-27 $\$ 645$

\section{Characterization of Materials}

Amorphous Silicon Technology

instructors: Robert A Street and Michael G. Hack

Monday, April 27

IC Fallure Mechanisms and Analytical Techniques

Instructor: Giorgio Riga

Thursday-Friday, April 30-May 1

Scanning Electron Microscopy: Applications to Electronic Materials and Devices

NeV !nstructor: Alton D. Romig. Jr.

TEM Specimen Preparation in the Physical Sciences

Instructor: Ronald M. Anderson

Monday afternoon-Tuesday, April 27-28
Characterization of Diamond Films

Instructors: Jeffrey T. Glass and Robert J. Nemanich

Sunday, Ap

Materials Research and Analysis Using In Situ and Ex Situ Spectroscopic Ellipsomatry

IInstructor: John A. Woolam

NeW Tuesday, April 28 ...

Preparation and Fabrication of Materials

Film and Coating Deposition Techniques instructor: Donald M. Mattox

Tuesday-Wednesday, April 28-29

Plasma Etching for Microelectronic Fabrication Instructor: G. Kenneth Herb Monday, April 27 ...

Materials and Processing Aspects of Advanced VLSI Assembly and Packaging

W ! Instructor: Shankara K. Prasad

Thursday-Saturday, April 30-May 2

Microwave Interactions with Dielectric Materials Instructors: Hal D. Kimrey and Magdy F. Iskander

Saturday-Sunday, April 25-26

Materials and Processes at the Leading Edge of Microlithography

NeW! Instructor: Gary N. Taylor

Friday, May 1

Film Formation, Adhesion, Surface Preparation, and Characterization of Thin Film Structures

Instructor: Donald M. Mattox

Saturday-Sunday, Aoril 25-26

Vapor Phase Synthesis of Powders and Films

G !nstructors: Toivo Kodas and Sotiris E. Pratsinis

NeW! Monday, April 27 ...

Fundamentals of Epitaxial Growth Techniques for Compound Semiconduclors Instructor: L. Raiph Dawson Saturday-Sunday, April 25-26

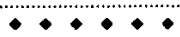

Tutorial Program

Introduction to Parallel Supercomputing in Malerial Sciance

Instructors: Jeftrey S. Nelson, Mark P. Sears and Steve J. Plimpton

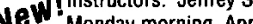

Special Fee Discounts:

- P-14 and F-01 - \$975 Total Fee; C-16 and C-12 - \$975 Total Fee

- Facilities registering three or more persons at the same time in one MRS short course receive a $20 \%$ discount for the third and all additional persons. 


\section{Spring Meeting Session Locator}

\begin{tabular}{|c|c|c|c|c|c|c|c|}
\hline \multirow[b]{2}{*}{ Activity } & \multirow[b]{2}{*}{ Location } & \multicolumn{3}{|c|}{ Monday, April 27} & \multicolumn{3}{|c|}{ Tuesday, April 28} \\
\hline & & a.m. & p.m. & eve & a.m. & p.m. & eve \\
\hline $\begin{array}{l}\text { A. Amorphous } \\
\text { Silicon }\end{array}$ & $\begin{array}{l}\text { Sunset } \\
A / B / C\end{array}$ & & & & $\begin{array}{l}\text { A1: Growth } \\
\text { A2: Defects }\end{array}$ & $\begin{array}{l}\text { A3: Transport } \\
\text { A4: Solar Cells }\end{array}$ & Posters \\
\hline $\begin{array}{l}\text { B. Chemical Surfaces } \\
\text { in Semiconductor } \\
\text { Growth }\end{array}$ & $\underset{E / F}{\text { Marina }}$ & B1: Surface Conditioning & B2: CVD and Oxidation & & $\begin{array}{l}\text { B3: UV- and Plasma- } \\
\text { Enhanced Processes }\end{array}$ & $\begin{array}{l}\text { B4: Compound } \\
\text { Semiconductors }\end{array}$ & Posters \\
\hline $\begin{array}{l}\text { C. Metallization/ } \\
\text { Processing for } \\
\text { Semiconductors }\end{array}$ & $\begin{array}{l}\text { Marina } \\
\text { A/B }\end{array}$ & $\begin{array}{l}\text { C1: Metallization - } \\
\text { Plenary Session }\end{array}$ & $\begin{array}{l}\text { C2: CVD and MOCVD } \\
\text { of Metals }\end{array}$ & & $\begin{array}{l}\text { C3: Silicides I- } \\
\text { Cobalt Silicides }\end{array}$ & $\begin{array}{l}\text { C4: Silicides II - } \\
\text { Commonn Systems }\end{array}$ & Posters \\
\hline $\begin{array}{l}\text { D. Space Charge } \\
\text { Effects in } \\
\text { Semiconductors }\end{array}$ & $\underset{\text { Hill }}{\text { Telegraph }}$ & & & & & & \\
\hline $\begin{array}{l}\text { E. Defect Engineering in } \\
\text { Semiconductors }\end{array}$ & $\begin{array}{l}\text { Sunset } \\
D / E / F\end{array}$ & $\begin{array}{l}\text { E1: Defects in Bulk } \\
\text { Crystals - I }\end{array}$ & $\begin{array}{c}\text { E2: Defects in Bulk } \\
\text { Crystals - II } \\
\text { E3: Defects in Thin Films }\end{array}$ & & $\begin{array}{c}\text { E4: Defect } \\
\text { Characterization }\end{array}$ & $\begin{array}{l}\text { E5: Hydrogen Interaction/ } \\
\text { Semiconductors }\end{array}$ & Posters \\
\hline $\begin{array}{l}\text { F. Heteroepitaxial } \\
\text { Growth }\end{array}$ & $\begin{array}{l}\text { Marina } \\
\text { C/D }\end{array}$ & & $\begin{array}{l}\text { F1: Surface Structure } \\
\text { and Reactions }\end{array}$ & & $\begin{array}{l}\text { F2: Nucleation and Thin } \\
\text { Film Evolution }\end{array}$ & F3: Novel Synthesis & \\
\hline $\begin{array}{l}\text { G. Electronic } \\
\text { Packaging }\end{array}$ & $\begin{array}{l}\text { Potrero } \\
\text { Hill }\end{array}$ & $\begin{array}{c}\text { G1: System and } \\
\text { Technology Overview } \\
\text { G2: Materials Development }\end{array}$ & $\begin{array}{l}\text { G3: Materials and } \\
\text { Processing }\end{array}$ & & $\begin{array}{c}\text { G4: Thermal/Mechanical } \\
\text { Properties }\end{array}$ & $\begin{array}{l}\text { G5: Thermal Stress, } \\
\text { Adhesion and Reliability }\end{array}$ & Posters \\
\hline $\begin{array}{l}\text { H. Materials } \\
\text { Reliability }\end{array}$ & $\underset{A}{\text { Pacific }}$ & & & & & & \\
\hline $\begin{array}{l}\text { la. Recycling of } \\
\text { Wood-Based Materials }\end{array}$ & $\begin{array}{l}\text { Pacific } \\
\text { B }\end{array}$ & $\begin{array}{l}\text { la1: General } \\
\text { la2: Fiber to Composites }\end{array}$ & la2: Fiber to Composites & & la2: Fiber to Composites & la3: Paper to Paper & \\
\hline $\begin{array}{l}\text { Ib. Materials for } \\
\text { Energy Technologies }\end{array}$ & $\underset{\mathrm{H}}{\text { Pacific }}$ & & & & & $\begin{array}{l}\text { Ib1/lc4: Membrane } \\
\text { Catalysis }\end{array}$ & \\
\hline $\begin{array}{l}\text { Ic. Materials for } \\
\text { Separation }\end{array}$ & $\underset{\mathrm{H}}{\mathrm{Pacific}}$ & $\begin{array}{l}\text { Ic1: Polymeric } \\
\text { Membranes }\end{array}$ & $\begin{array}{l}\text { Ic2: Ceramic Membrane } \\
\text { Prep and } \\
\text { Characterization } \\
\end{array}$ & & $\begin{array}{l}\text { Ic3: Ceramic Membranes } \\
\text { for Gas Separations }\end{array}$ & $\begin{array}{l}\text { Ic4/Ib1: Membrane } \\
\text { Catalysis }\end{array}$ & \\
\hline $\begin{array}{l}\text { J. Art and } \\
\text { Archaeology }\end{array}$ & $\begin{array}{l}\text { Salon } \\
\text { A1 }\end{array}$ & & $\begin{array}{c}\text { J1: Treatment, } \\
\text { Deterioration and } \\
\text { Structure }\end{array}$ & & $\begin{array}{l}\text { J2: Technical Analysis: } \\
\text { Structure and Composition }\end{array}$ & J3: Behavior of Materials & Posters \\
\hline $\begin{array}{l}\text { K. Materials Modif. by } \\
\text { Energetic Atoms } \\
\text { and lons }\end{array}$ & $\begin{array}{l}\text { Salon } \\
\text { B3 }\end{array}$ & & & & $\begin{array}{l}\text { K1: Reactive lon Etching } \\
\text { and Surface Damage }\end{array}$ & $\begin{array}{l}\text { K2: Plenary Session/ } \\
\text { Film Growth }\end{array}$ & \\
\hline $\begin{array}{l}\text { L. Microwave } \\
\text { Processing }\end{array}$ & $\begin{array}{l}\text { Salon } \\
\mathrm{C} 1\end{array}$ & L1: Overview/Plenary & $\begin{array}{c}\text { L2: Numerical Modeling } \\
\text { Techniques } \\
\text { L3: Microwave/Plasma } \\
\text { Processing }\end{array}$ & & \begin{tabular}{|c|} 
L4: Microwave Processing \\
System Design \\
L5: Microwave NDE \\
Techniques \\
\end{tabular} & $\begin{array}{l}\text { L6: Dielectric Properties } \\
\text { and Measurements }\end{array}$ & Posters \\
\hline $\begin{array}{l}\text { M. Novel Forms } \\
\text { of Carbon }\end{array}$ & $\begin{array}{l}\text { Salon } \\
\text { A2 }\end{array}$ & M1: Foams and Aerogels & $\begin{array}{l}\text { M2: Surfaces, Alloys, and } \\
\text { Mixed Phase Materials }\end{array}$ & & $\begin{array}{c}\text { M3/P1: Fullerenes } \\
\text { Fullerrides and Fulleroids I }\end{array}$ & $\begin{array}{l}\text { M4: Fullerenes, Fullerrides } \\
\text { and Fulleroids II }\end{array}$ & Posters \\
\hline $\begin{array}{l}\text { N. Better Ceramics } \\
\text { through Chemistry }\end{array}$ & $\begin{array}{l}\text { Salon } \\
\text { B2 }\end{array}$ & $\begin{array}{l}\text { N1: Molecular Routes to } \\
\text { Ceramic Materials }\end{array}$ & $\begin{array}{l}\text { N1: Molecular Routes to } \\
\text { Ceramic Materials }\end{array}$ & $\begin{array}{l}\text { N2: Hybrid } \\
\text { Organic/ } \\
\text { Inorganic } \\
\text { Materials } \\
\end{array}$ & $\begin{array}{l}\text { N3: Metal Nitrides and } \\
\text { and Calcogenides }\end{array}$ & $\begin{array}{l}\text { N3: Metal Carbides, } \\
\text { Borides and Nitrides }\end{array}$ & $\begin{array}{l}\text { N4/P3: Oxide } \\
\text { Ceramics }\end{array}$ \\
\hline $\begin{array}{l}\text { 0. Metal and } \\
\text { Semiconductor } \\
\text { Clusters and Colloids }\end{array}$ & $\begin{array}{l}\text { Salon } \\
\text { A3 }\end{array}$ & $\begin{array}{l}\text { 01: Semiconductors } \\
\text { Colloid/Cluster Synthesis }\end{array}$ & $\begin{array}{l}\text { 02: Stability of } \\
\text { Semiconductor Clusters }\end{array}$ & & $\begin{array}{l}\text { 03: Metal Cluster Synthesis } \\
\text { and Characterization }\end{array}$ & $\begin{array}{l}\text { 04: Molecular Metal } \\
\text { Clusters }\end{array}$ & \\
\hline $\begin{array}{l}\text { P. Aerosol } \\
\text { Precursors }\end{array}$ & $\begin{array}{l}\text { Salon } \\
\text { A2 }\end{array}$ & & & & $\begin{array}{c}\mathrm{P} 1 / \mathrm{M} 3 \text { : Fullerenes } \\
\text { Fullerrides and Fulleroids I }\end{array}$ & $\begin{array}{l}\text { P2: Nanophase Particle } \\
\text { Generation } \\
\text { (Pacific I) }\end{array}$ & $\begin{array}{l}\text { P3/N4: Oxide } \\
\text { Ceramics } \\
\text { (Salon B2) } \\
\end{array}$ \\
\hline $\begin{array}{l}\text { a. Intermetallic } \\
\text { Matrix Composites }\end{array}$ & $\begin{array}{l}\text { Salon } \\
\text { B1 }\end{array}$ & $\begin{array}{l}\text { Q1: MoSi2 Matrix } \\
\text { Composites }\end{array}$ & Q2: Modeling and Testing & & Q3: In-Situ Composites & $\begin{array}{l}\text { Q4: New Processies and } \\
\text { Materials }\end{array}$ & \\
\hline $\begin{array}{l}\text { R. Submicron } \\
\text { Multiphase Materials }\end{array}$ & $\underset{J}{\text { Pacific }}$ & & & & $\begin{array}{l}\text { R1: Polymer/Polymer } \\
\text { Composites }\end{array}$ & $\begin{array}{l}\text { R2: Polymer/Polymer } \\
\text { Composites }\end{array}$ & \\
\hline $\begin{array}{l}\text { S. Layered } \\
\text { Superconductors }\end{array}$ & $\begin{array}{l}\text { Salon } \\
\text { C2 }\end{array}$ & S1: Fundamentals I & S2: Fundamentals II & & $\begin{array}{l}\text { S3: Layer-By-Layer Film } \\
\text { Growth }\end{array}$ & $\begin{array}{l}\text { S4: Thin Films: Deposition } \\
\text { and Characterization }\end{array}$ & \\
\hline $\begin{array}{l}\text { T. Defects in Crystalline } \\
\text { Electronic Oxides }\end{array}$ & $\begin{array}{l}\text { Salon } \\
\text { A3 }\end{array}$ & & & & & & \\
\hline $\begin{array}{l}\text { U/Y. "Smart"' Materials/ } \\
\text { Micro-Electro-Mechan- } \\
\text { ical Systems }\end{array}$ & $\begin{array}{l}\text { Salon } \\
\text { C3 }\end{array}$ & & & & $\begin{array}{l}\text { U1/Y1: Ferroelectrics I } \\
\text { U2/Y2: Ferroelectrics - II }\end{array}$ & \begin{tabular}{|c|} 
U3/Y3: Polycrystalline \\
Silicon \\
U4/Y4: Optical Sensors and \\
Systems \\
\end{tabular} & \\
\hline $\begin{array}{l}\text { V. Macromolecular } \\
\text { Host-Guest Complexes }\end{array}$ & $\begin{array}{c}\text { Telegraph } \\
\text { Hill }\end{array}$ & $\begin{array}{l}\text { V1: Photoconductors and } \\
\text { Optical Storage Materials }\end{array}$ & $\begin{array}{l}\text { V2: Coordination } \\
\text { Compounds }\end{array}$ & & $\begin{array}{c}\text { V3: Organic Photorefractive } \\
\text { Materials }\end{array}$ & $\begin{array}{l}\text { V4: Nonlinear Optical and } \\
\text { Optoelectronic Properties } \\
\end{array}$ & \\
\hline $\begin{array}{l}\text { W. Computational } \\
\text { Methods }\end{array}$ & $\begin{array}{l}\text { Nob } \\
\text { Hill }\end{array}$ & & W1: Modeling Techniques & & W2: Polymers & $\begin{array}{l}\text { W3: Ceramics and } \\
\text { Semiconductors }\end{array}$ & \\
\hline $\begin{array}{l}\text { X. Frontiers of } \\
\text { Materials Research }\end{array}$ & $\begin{array}{l}\text { Sunset } \\
A / B / C\end{array}$ & & $\begin{array}{c}\mathrm{x}_{1} \\
12: 05-1: 25 \mathrm{p} . \mathrm{m} .\end{array}$ & & & $\begin{array}{c}\mathrm{X} 2 \\
12: 05-1: 25 \mathrm{p} . \mathrm{m} .\end{array}$ & \\
\hline
\end{tabular}




\begin{tabular}{|c|c|c|c|c|c|c|c|}
\hline \multicolumn{3}{|c|}{ Wednesday, April 29} & \multicolumn{3}{|c|}{ Thursday, April 30} & \multicolumn{2}{|c|}{ Friday, May 1} \\
\hline a.m. & p.m. & eve & a.m. & p.m. & eve & a.m. & p.m. \\
\hline $\begin{array}{l}\text { A6: Thin Film Transistors } \\
\text { A7: Structure }\end{array}$ & $\begin{array}{l}\text { A8: Stability I } \\
\text { A9: Stability II }\end{array}$ & & $\begin{array}{l}\text { A10: Alloys and Multilayers } \\
\text { A11: Radiation Detectors }\end{array}$ & $\begin{array}{l}\text { S12: Electronic Properties } \\
\text { A13: Novel Devices }\end{array}$ & Posters & $\begin{array}{l}\text { A15: Image Sensors } \\
\text { A16: Metastability }\end{array}$ & \\
\hline B6: HF Cleaning & B7: Epitaxial Growth & & & & & & \\
\hline \multirow[t]{2}{*}{$\begin{array}{l}\text { C6: Schottky and Ohmic } \\
\text { Contacts to GaAs }\end{array}$} & $\begin{array}{l}\text { C7: Contacts to InP and } \\
\text { Related Materials }\end{array}$ & Posters & $\begin{array}{l}\text { C9/H3: Metallization } \\
\text { Schemes }\end{array}$ & $\begin{array}{l}\text { C10: Metallization Schemes } \\
\text { c11: Contacts to Multilayers }\end{array}$ & & $\begin{array}{l}\text { C12: Contacts to Insulators } \\
\text { and Dielectric Layers }\end{array}$ & \\
\hline & D1: Electro-0ptics & & D2: Silicon and Interfaces & D3: Photoconductivity & Posters & D5: Defects & $\begin{array}{l}\text { D6: Optical Materials and } \\
\text { Applications }\end{array}$ \\
\hline $\begin{array}{l}\text { E7: Defect Properties and } \\
\text { Reactions }\end{array}$ & $\begin{array}{l}\text { E8: Defects Induced by } \\
\text { Processing }\end{array}$ & & E9: Defects in Devices & $\begin{array}{l}\text { E10: Quantum Wells, } \\
\text { superlattices and Interfaces }\end{array}$ & Posters & $\begin{array}{c}\text { E12: Gettering and Related } \\
\text { Phenomena }\end{array}$ & E13: Ion Implantation \\
\hline F4: Internal Surfaces & $\begin{array}{l}\text { F5: Electrical, Optical and } \\
\text { Magnetic Properties }\end{array}$ & Posters & F7: Strain Relief - I & F8: Strain Relief - II & & & \\
\hline G7: Low-End Packaging & $\begin{array}{l}\text { G8: Optical Interconnects: } \\
\text { Systems and Applications }\end{array}$ & & G9: Optical Interconnects & $\begin{array}{l}\text { G10: Optical Interconnects: } \\
\text { Manufacturing Issues }\end{array}$ & & & \\
\hline $\begin{array}{l}\text { H1: Stress and } \\
\text { Electromigration/Modeling }\end{array}$ & $\begin{array}{l}\text { H2: Microstructure and } \\
\text { Electronics }\end{array}$ & & $\begin{array}{l}\text { H3/C9: Interconnects, } \\
\text { Wiring, and Packaging } \\
\text { (Marina A/B) }\end{array}$ & $\begin{array}{l}\text { H4: Oxide and Device } \\
\text { Reliability } \\
\text { (Marina EF) }\end{array}$ & & $\begin{array}{l}\text { H5: Analytical Techniques } \\
\text { (Marina E/F) }\end{array}$ & \\
\hline la3: Paper to Paper & $\begin{array}{l}\text { Ia4: Roundtable } \\
\text { Discussion }\end{array}$ & & & & & & \\
\hline $\begin{array}{l}\text { Ib2: Catalysis and } \\
\text { Separations }\end{array}$ & $\begin{array}{l}\text { Ib3: Composite and Alloy } \\
\text { Materials }\end{array}$ & & Ib4: Electronic Applications & & & & \\
\hline $\begin{array}{l}\text { J5: Cultural Heritage in } \\
\text { Conflicts }\end{array}$ & $\begin{array}{l}\text { J5: Cultural Heritage in } \\
\text { Conflicts }\end{array}$ & & $\begin{array}{l}\text { J6: Technical Analysis of } \\
\text { Materials }\end{array}$ & J7: Metallurgy & & J8: Glass and Ceramics & J8: Glass and Ceramics \\
\hline K3: Film Growth & K4: Heteroepitaxy & Posters & $\begin{array}{l}\text { K6: Ion Implanation of } \\
\text { Semiconductors } \\
\text { K7: lon Beam Processing }\end{array}$ & K8: Ion Beam Modification & & & \\
\hline $\begin{array}{l}\text { L6: Dielectric Properties } \\
\text { and Measurements } \\
\text { L8: Microwave/Materials } \\
\text { Interactions }\end{array}$ & $\begin{array}{c}\text { L9: Microwave Processing } \\
\text { of Ceramics }\end{array}$ & & $\begin{array}{l}\text { L9: Microwave Processing } \\
\text { of Ceramics } \\
\text { L10: Microwave Processing } \\
\text { of Hazardous Wastes }\end{array}$ & $\begin{array}{c}\text { L11: Microwave Processing } \\
\text { of Polymers and } \\
\text { Composites }\end{array}$ & & $\begin{array}{l}\text { L12: Millimeter Wave } \\
\text { Processing }\end{array}$ & \\
\hline $\begin{array}{l}\text { M6: Fullerenes, Fullerides } \\
\text { and Fulleroids III }\end{array}$ & $\begin{array}{l}\text { M7: Fullerenes, Fullerides } \\
\text { and Fulleriods IV }\end{array}$ & & M8: Diamond I & M9: Diamond II & & M10: Diamond-Like Carbon & \\
\hline $\begin{array}{l}\text { N5: Particulate and } \\
\text { Polymeric Sols }\end{array}$ & $\begin{array}{l}\text { N6: Aging, Drying and } \\
\text { Consolidation of Gels }\end{array}$ & Posters & N10: Ceramic Thin Films & N10: Ceramic Thin Films & Posters & $\begin{array}{l}\text { N15: Dense and Porous } \\
\text { Coatings }\end{array}$ & N16: Composite Ceramics \\
\hline 05: Theoretical Aspects & $\begin{array}{l}\text { 06: Metal Colloids and } \\
\text { Nanophase Materials }\end{array}$ & & & & & & \\
\hline $\begin{array}{l}\text { P4: Powder Synthesis by } \\
\text { Aerosol Processes } \\
\text { (Pacific I) }\end{array}$ & $\begin{array}{l}\text { P5: Novel Techniques and } \\
\text { Materials } \\
\text { (Pacific I) }\end{array}$ & & $\begin{array}{l}\text { P6/W6: Deposition and } \\
\text { Clusters } \\
\text { (Nob Hill) }\end{array}$ & & & & \\
\hline $\begin{array}{l}\text { 05: Nickle Aluminide Matrix } \\
\text { Composites }\end{array}$ & $\times \quad \begin{array}{l}\text { Q6: Titanium Aluminide } \\
\text { Composites I }\end{array}$ & & $\begin{array}{l}\text { Q7: Titanium Aluminide } \\
\text { Composites II }\end{array}$ & & & & \\
\hline $\begin{array}{c}\text { R3: Ceramic } \\
\text { (inorganic)/Polymer } \\
\text { Composites }\end{array}$ & $\begin{array}{c}\text { R4: Ceramic } \\
\text { (Inoranaic/)/Polymer } \\
\text { (Organic) Composites } \\
\text { R5: Ceramic (lnorganic) } \\
\text { Composites }\end{array}$ & & $\begin{array}{l}\text { R6: Ceramic (Inorganic) } \\
\text { Composites }\end{array}$ & & & & \\
\hline \multirow[t]{2}{*}{$\begin{array}{l}\text { S5: Vortex Dynamics and } \\
\text { Flux Pinning }\end{array}$} & $\begin{array}{c}\text { S6: Microstructures, } \\
\text { Growth Kinetics and Critical } \\
\text { Currents }\end{array}$ & Posters & $\begin{array}{l}\text { S8: Applications and } \\
\text { Related Topics }\end{array}$ & $\begin{array}{l}\text { S9: Bulk Processing and } \\
\text { Properties I }\end{array}$ & Posters & $\begin{array}{l}\text { S11: Bulk Processing and } \\
\text { Properties II }\end{array}$ & \\
\hline & & & T1: Oxide Defects & $\mathrm{T} 2: \mathrm{ZnO}$ and Others & & T3: Perovskites & T4: HTC Superconductors \\
\hline $\begin{array}{l}\text { U5/Y5: Thin Film Shape } \\
\text { Memory Effect NiTi } \\
\text { U6/Y6: Bulk "Smart" } \\
\text { Structures - } 1\end{array}$ & $\begin{array}{l}\text { U7/Y7: Biological and } \\
\text { Chemical Sensing } \\
\text { Applications } \\
\text { U8/r8: Bulk "Smart" } \\
\text { Materials - } 11\end{array}$ & & $\begin{array}{l}\text { U9/Y9: Thermomechanical } \\
\text { Applications } \\
\text { U10/r10: Materials } \\
\text { Characterization - I }\end{array}$ & $\begin{array}{l}\text { U11/ } Y 11: \text { Materials } \\
\text { Characterization - II } \\
\text { U12/Y12: Alternative } \\
\text { Materials and Process } \\
\text { Modeling }\end{array}$ & & & \\
\hline \multirow[t]{2}{*}{ W4: Mechancial Properties } & $\begin{array}{c}\text { W5: Structure of Surfaces } \\
\text { and Interfaces }\end{array}$ & & $\begin{array}{l}\text { W6/P6: Deposition and } \\
\text { Clusters }\end{array}$ & $\begin{array}{l}\text { W7: Materials Processing } \\
\text { and Dynamics }\end{array}$ & & $\begin{array}{l}\text { W8: Structure and } \\
\text { Properties I }\end{array}$ & $\begin{array}{l}\text { W9: Structure and } \\
\text { Properties II }\end{array}$ \\
\hline & $\begin{array}{c}X 3 \\
12: 05-1: 25 \text { p.m. }\end{array}$ & & & $\begin{array}{l}\text { X4: Advanced Materials } \\
\text { Initiative Forum }\end{array}$ & & & $\begin{array}{c}X 5 \\
12: 05-1: 25 p . m .\end{array}$ \\
\hline
\end{tabular}

